J. DIFFERENTIAL GEOMETRY

45 (1997) 53-73

\title{
RIEMANN-ROCH FOR TORIC ORBIFOLDS
}

VICTOR GUILLEMIN

\section{Introduction}

Let $\alpha_{1}, \ldots, \alpha_{d}$ and $\mu$ be elements of the integer lattice, $\mathbf{Z}^{n}$, and let $N(\mu)$ be the number of solutions, $k=\left(k_{1}, \ldots, k_{d}\right)$, of the equation

$$
k_{1} \alpha_{1}+\ldots+k_{d} \alpha_{d}=\mu,
$$

the $k_{i}$ 's being non-negative integers. For this equation to be well-posed we will assume that the $\alpha_{i}$ 's lie in a fixed open half-space. In other words: for all $i, \xi\left(\alpha_{i}\right)>0$, for some $\xi \in\left(\mathbf{R}^{n}\right)^{*}$. (Otherwise, for every $\mu$ for which (1.1) admits a solution it will admit an infinite number of solutions!) Also, in order for (1.1) to be solvable, $\mu$ has to be contained in the lattice generated by the $\alpha_{i}$ 's, and, with no essential loss of generality, we can assume that this lattice is $\mathbf{Z}^{n}$ itself.

For every subset, $I$, of $\{1, \ldots, d\}$ let $\mathbf{R}^{I}$ be the subspace of $\mathbf{R}^{n}$ spanned by those $\alpha_{i}$ 's for which $i$ is in $I$. We will say that $\mu$ is in general position with respect to $\alpha_{1}, \ldots, \alpha_{d}$ if $\mu \in \mathbf{R}^{I} \leftrightarrow \mathbf{R}^{I}=\mathbf{R}^{n}$. (Thus the elements of $\mathbf{R}^{I}$ are not in general position with respect to $\alpha_{1}$, $\ldots, \alpha_{d}$ if $\mathbf{R}^{I}$ is a proper subspace of $\mathbf{R}^{n}$.)

Let us consider the real analogue of (1.1):

$$
s_{1} \alpha_{1}+\ldots+s_{d} \alpha_{d}=\mu+\epsilon \quad, \quad \epsilon \in \mathbf{R}^{n},
$$

the $s_{i}$ 's being non-negative real numbers. The set of solutions, $s$, of this equation is a convex polytope in $\mathbf{R}^{d}$. We will denote this polytope by $\Delta_{\mu+\epsilon}$ and its $I$-th face:

$$
\Delta_{\mu+\epsilon}^{I}=\left\{s=\left(s_{1}, \ldots, s_{d}\right) \in \Delta_{\mu+\epsilon}, s_{i}=0 \text { for } i \in I\right\}
$$

by $\Delta_{\mu+\epsilon}^{I}$. We claim:

Received December 26 1995. Author supported by NSF grant DMS 890771. 
Theorem 1.1. If $\mu$ is in general position with respect to $\alpha_{1}, \ldots$, $\alpha_{d}$ the volume of $\Delta_{\mu+\epsilon}^{I}$ is, for $\epsilon$ small, a polynomial of degree $d-n-|I|$ in $\epsilon$.

Denoting this polynomial by $v^{I}(\epsilon)$, we will prove below the following:

Theorem 1.2. For $\mu$ in general position with respect to $\alpha_{1}, \ldots, \alpha_{d}$,

$$
N(\mu)=\sum_{I} \mathcal{T}_{I}\left(\frac{\partial}{\partial \epsilon}\right) v^{I}(\epsilon)
$$

evaluated at $\epsilon=0$, the $\mathcal{T}_{I}$ 's being constant coefficient differential operators of "infinite order" (explicit formulas for which will be given in $§ 3$. See (3.28)-(3.29).)

For instance for $I=\phi, \mathcal{T}_{I}$ is the Todd operator

$$
\prod_{i=1}^{d} \frac{\partial}{\partial \epsilon_{i}}\left(1-\exp \left(-\frac{\partial}{\partial \epsilon_{i}}\right)\right)^{-1} .
$$

We will break the proof of (1.4) into two parts:

1. Let $T$ be the standard $n$-torus and let $\rho$ be the linear action of $T$ on $\mathbf{C}^{d}$ defined by

$$
\rho(\exp x) z=\left(e^{2 \pi i \alpha_{1} \cdot x} z_{1}, \ldots, e^{2 \pi i \alpha_{d} \cdot x} z_{d}\right)
$$

$x$ being in $\mathbf{R}^{n}$ and $\exp x$ its image in $T$. If we equip $\mathbf{C}^{d}$ with the symplectic form

$$
\omega=\sqrt{-1} \sum_{r=1}^{d} d z_{r} \wedge d \bar{z}_{r}
$$

$\rho$ becomes a Hamiltonian action, and its moment map is

$$
\Phi(x)=\sum_{r=1}^{d}\left|z_{r}\right|^{2} \alpha_{r} .
$$

If $\mu$ is in general position with respect to $\alpha_{1}, \ldots, \alpha_{d}, \mu$ is a regular value of $\Phi$; so the reduced space

$$
X=\Phi^{-1}(\mu) / T
$$

is a symplectic orbifold. Let $\mathcal{B}_{d}$ be the Bargmann space, i.e., the space of holomorphic functions on $\mathbf{C}^{d}$ which are $L^{2}$-integrable with respect to 
the Gaussian measure, $(-i)^{d} e^{-|z|^{2}} d z d \bar{z}$. By a result of Meinrenken [18] and Vergne [25] the multiplicity with which $\mu$ occurs as a weight of the representation of the group, $T$, on $\mathcal{B}_{d}$ is equal to the Kawasaki-RiemannRoch number of $X$. On the other hand the elements of the Bargmann space which transform according to the weight, $\mu$, are precisely the monomials

$$
z^{k}=z_{1}^{k_{1}} \ldots z_{d}^{k_{d}}
$$

for which $k$ is a solution of (1.1). Thus $N(\mu)$ is equal to the KawasakiRiemann-Roch number of $X$.

2. Hence it suffices to show that the Kawasaki-Riemann-Roch number of $X$ is given by an expression of the form (1.4). This we will do by using the fact that the volume of the $I$-th stratum of the reduced space

$$
X_{\epsilon}=\Phi^{-1}(\mu+\epsilon) / T
$$

is equal, on one hand, to the volume of $\Delta_{\mu+\epsilon}^{I}$; and, on the other hand, by the Duistermaat-Heckman theorem, is equal to the integral over the $I$-th stratum of $X$ of $\exp \left(\omega+\sum \epsilon_{i} c_{i}\right), \omega$ being the reduced symplectic form and the $c_{i}$ 's being the Chern classes of $X$. From this identity it follows that every "mixed characteristic number" of $X$

$$
\sum_{I} \int_{X^{I}} \exp [\omega] \mathcal{T}_{I}\left(c_{1}, \ldots, c_{d}\right)
$$

(for example, the Kawasaki-Riemann-Roch number) can be written as an expression of the form (1.4).

Theorem 1.2 has the following interesting generalization.

Theorem 1.3. Let $P(s)$ be a polynomial in $s_{1}, \ldots, s_{d}$. Then the sum

$$
\sum_{k} P(k)
$$

over the set of solutions, $k$, of (1.1) is equal to

$$
\sum_{I} \mathcal{T}_{I}\left(\frac{\partial}{\partial \epsilon}\right) \int_{\Delta_{\mu+\epsilon}^{I}} P\left(s_{1}, \ldots, s_{d}\right) d s
$$

evaluated at $\epsilon=0$. 
The proof of this is similar to the proof we have just sketched: For $\xi \in \mathbf{R}^{d}$ the sum

$$
\sum e^{i \xi \cdot x}
$$

over the solutions, $k$, of (1.1) is equal to the equivariant KawasakiRiemann-Roch number of $X$; and we will show in $\S 4$ that this is equal to

$$
\sum \mathcal{T}_{I}\left(\frac{\partial}{\partial \epsilon}\right) \int_{\Delta_{\mu+\epsilon}^{I}} e^{i \xi \cdot s} d s
$$

evaluated at $\epsilon=0$. (The identity (1.13), for the polynomial, $s^{\beta}$, can be proved by differentiating both sides of this identity by $D_{\xi}^{\beta}$ and setting $\xi=0$.)

Finally we will describe what happens if $\mu$ is not in general position with respect to the $\alpha_{i}$ 's. In this case one can define the Kawasaki Riemann-Roch number of the reduced space (1.9) to be equal to

$$
\sum_{I} \int_{X_{\epsilon}^{I}} \exp \left(\omega_{\epsilon}-\sum \epsilon_{i} c_{i}\right) \mathcal{T}_{I}\left(c_{1}, \ldots, c_{d}\right)
$$

with $\epsilon=\sum \epsilon_{i} \alpha_{i}, \mu+\epsilon$ being in general position with respect to the $\alpha_{i}$ 's, $\mathcal{T}_{I}$ being given, as above, by (3.28), and $\omega_{\epsilon}$ being the reduced symplectic form on $X_{\epsilon}$. Using the Duistermaat-Heckman theorem it is easy to see that the expression (1.16) does not change if one replaces $\epsilon$ by $\epsilon^{\prime}$ providing all points on the line segment joining $\mu+\epsilon$ to $\mu+\epsilon^{\prime}$ are in general position with respect to the $\alpha_{i}$ 's. However, we claim that more is true: Let $C$ be the cone

$$
\left\{\sum s_{i} \alpha_{i}, s_{i} \geq 0\right\},
$$

i.e., let $C$ be the image of the moment map (1.8).

Theorem 1.4. If $\mu+\epsilon$ is in $C$ and $\epsilon$ is sufficiently small, then the Riemann-Roch number (1.16) is independent of $\epsilon$ and is equal to $N(\mu)$.

This theorem follows from a result of Meinrenken-Sjamaar which we will describe in section 5. Noting that (1.16) is a mixed characteristic number of $X_{\epsilon}$ of the type (1.12), we obtain the following corollary:

Theorem 1.5. Let $\epsilon$ tend to zero along any ray

$$
\mu+\epsilon=\mu+t \nu, \quad 0<t<t_{0},
$$


all points of which lie in $C$ and are in general position with respect to the $\alpha_{i}$ 's. Then

$$
N(\mu)=\operatorname{Lim}_{\epsilon \rightarrow 0} \sum \mathcal{T}_{I}\left(\frac{\partial}{\partial \epsilon}\right) v_{I}(\epsilon)
$$

Meinrenken and Sjamaar have also proved an equivariant version of the result we just cited, from which one gets an analogous formula for the sum (1.13).

\section{Comments and acknowledgments}

Theorems 1.3 and 1.5 have been proved independently, using different methods from ours, by Michel Brion and Michele Vergne [3]. Sylvain Cappell and Julius Shaneson have also announced in [4] a result similar to (1.13) (not, however, involving the dilated polytope, $\Delta_{\mu+\epsilon}$ ). An important special case of (1.15) was discovered by Askold Khovanskii in the late nineteen-eighties. He showed that if the toric variety (1.9) is non-singular, the sum (1.12) is equal to the leading term in (1.13). See [14] and [13]. The fact that the number of solutions of (1.1) is the Riemann-Roch invariant of a toric variety has been known for a long time (see, for instance, [5]), and has been used by many people, notably by Brion [2], Morelli [20], Pommersheim [21] and Sturmfels [24] to obtain various kinds of explicit formulas for $N(\mu)$. We also appear not to have been the first persons to have noticed that Duistermaat-Heckman can be useful for computing this invariant. See Brion, loc. cit., page 662 , line $12^{-}$.

The results reported on below are part of a project which we are involved in with Viktor Ginzburg and Yael Karshon. The goal of this project is a "hands-on" proof of Theorem 2.3 of $\S 2$ (the MeinrenkenVergne theorem) by cobordism methods.

\section{Kawasaki-Riemann-Roch}

Let $M$ be a manifold, $G$ a compact connected Lie group and $\tau: G \times$ $M \rightarrow M$ an action of $G$ on $M . \tau$ is locally free if, for every point, $m$, the stabilizer group of $m$ is a finite subgroup of $G$. If $\tau$ is an action of this type the quotient space

$$
X=: M / G
$$

is an orbifold. A theorem of Satake [22] says that the converse is true: every orbifold has a presentation of the form (2.1). For the orbifolds 
we will be concerned with in this article $G$ can be taken to be abelian, i.e., to be the standard $n$-torus. We will also assume below that $X$ and $M$ are compact and that $\tau$ is faithful (i.e., is an injective map of $G$ into $\operatorname{Diff}(M)$.) For a summary of basic facts about orbifolds we refer to [22]. One of the facts we will need is that an orbifold has an intrinsic stratification and that to each stratum one can assign, in an intrinsic way, the "structure group" of that stratum. In terms of the presentation (2.1) the description of this stratification is the following: Since $M$ is compact there are only a finite number of subgroups of $G$ which can occur as isotropy groups of points. For each of these groups, $\Gamma$, let $Z$ be a connected component of $M^{\Gamma}$ and let $Z^{0}$ be the subset of $Z$ consisting of those points for which the stabilizer group is exactly $\Gamma$. $Z$ is a compact submanifold of $M$, and $Z^{0}$ an open dense subset of $Z$. Moreover both $Z$ and $Z^{0}$ are $G$-invariant, and the quotient group, $G / \Gamma=G_{\Gamma}$, acts in a locally free fashion on $Z$ and acts freely on $Z^{0}$; so the quotient space

$$
F=Z / G_{\Gamma}
$$

is a suborbifold of $X$, and the quotient space

$$
F^{0}=Z^{0} / G_{\Gamma}
$$

is an open dense submanifold of $F$.

Definition 2.1. The sets, $F^{0}$, are the strata of the orbifold stratification of $X$, and the structure group of $F^{0}$ is $\Gamma$.

The set of strata forms a poset (partially ordered set) and so does the set of subgroups of $G$ which occur as isotropy groups; however, the correspondence

$$
\text { stratum } \rightarrow \text { structure group }
$$

inverts the ordering in these posets: If $F^{0}$ is contained in the closure of the stratum $F_{1}^{0}$ the structure group of $F_{1}^{0}$ is contained in $\Gamma$. We will denote by $\Gamma^{\#}$ the set of all elements, $g \in \Gamma$, having the property that $g$ is not contained in the structure group of any stratum whose closure contains $F^{0}$.

The orbifolds we will be interested in here are symplectic orbifolds, and in terms of the presentation (2.1), a symplectic form on $X$ can be defined as follows: A $k$-form, $\omega \in \Omega(M)$, is basic if

$$
\iota\left(\xi_{M}\right) \omega=\iota\left(\xi_{M}\right) d \omega=0
$$


for every $\xi \in \mathfrak{g}$, and a symplectic form is a basic two-form, $\omega$, with the property that, for every point, $m$, the annihilator of $\omega$ at $m$ :

$$
\left\{v \in T_{m} M, \iota(v) \omega=0\right\}
$$

is the tangent space to $G \cdot m$ at $m$. Notice that if $\omega$ has this property, and $F^{0}$ is a stratum of $X$ with the presentation (2.3), then the restriction of $\omega$ to $Z^{0}$ also has this property; so it is the pull-back to $Z^{0}$ of a symplectic form on $F^{0}$. Thus each stratum, $F^{0}$, is a symplectic manifold.

Let $T^{H}$ be the "horizontal component" of the tangent bundle of $M$, i.e., at each point, $m$, let $T_{m}^{H}$ be the quotient

$$
T_{m} M / T_{m}(G \cdot m)
$$

From a symplectic structure on $X$ one gets a symplectic structure on this vector bundle i.e., a symplectic bilinear form on each fiber, and this implies that this vector bundle can be given a $G$-invariant complex structure which is compatible with this structure. Moreover, though this complex structure will not be unique, it will be unique up to isotopy; so in particular the Chern classes of $T^{H}$ will be well-defined independent of the choice of this structure.

One other assumption which we will make is that the symplectic structure on $X$ is "pre-quantizable". In terms of (2.1) this means that there exists a line bundle, $\mathbf{L} \rightarrow M$, an action

$$
\tau_{\mathbf{L}}: G \rightarrow \text { automorphisms of } \mathbf{L}
$$

which is compatible with $\tau$ and a $G$-invariant connection on $\mathbf{L}$ whose curvature form is $\omega$.

By now we have listed all the prerequisites needed for our definition of Kawasaki-Riemann-Roch; however, it will be convenient in addition to make the following assumption about $T^{H}$. Let $\widetilde{\mathbf{C}}^{\ell}$ be the trivial vector bundle with fiber $\mathbf{C}^{\ell}$. We will assume that there is a $G$-invariant splitting

$$
T^{H} \oplus \widetilde{\mathbf{C}}^{\ell}=\mathbf{L}_{1} \oplus \ldots \oplus \mathbf{L}_{q}
$$

each of the summands on the left being a complex line bundle. At first glance this might seem to be an unduly restrictive assumption; but, fortunately, the orbifolds which we will discuss in $\S 3$ will have this property. Moreover, that deux ex machina, the "splitting principle" 
says that one can always operate as if such a splitting did exist (see $[1, \S 21])$.

The Kawasaki-Riemann-Roch number of $X$ is by definition an "invariant of stratified spaces", i.e., it can be written as a sum over the strata of $X$ :

$$
\sum \operatorname{KRR}\left(F^{0}\right)
$$

where $\operatorname{KRR}\left(F^{0}\right)$ is the "Kawasaki-Riemann-Roch number" of the stratum, $F^{0}$. To define this we will assume that $F^{0}$ has a presentation of the form (2.3), and its closure a presentation of the form (2.2). Let

$$
\widetilde{c}\left(\mathbf{L}_{i}\right) \in H_{G}^{2}(M, \mathbf{C})
$$

be the equivariant Chern class of $\mathbf{L}_{i}$, and

$$
\widetilde{c}(\mathbf{L}) \in H_{G}^{2}(M, \mathbf{C})
$$

the equivariant Chern class of the pre-quantum line bundle, L. Let $\pi$ be the projection of $M$ onto the quotient space, $M / G$. By (2.1) this induces a contravariant map on cohomology

$$
\pi^{\#}: H^{*}(X, \mathbf{C}) \rightarrow H_{G}^{*}(M, \mathbf{C}),
$$

and, by the Cartan theorem, this is a bijection. Let

$$
c\left(\mathbf{L}_{i}\right)=\left(\pi^{\#}\right)^{-1} \widetilde{c}\left(\mathbf{L}_{i}\right) \text { and } c(\mathbf{L})=\left(\pi^{\#}\right)^{-1} \widetilde{c}(\mathbf{L}),
$$

and let $\iota$ be the inclusion map of $Z$ into $M$. From (2.4) we get a splitting of vector bundles (over $Z$ ):

$$
\iota^{*} T^{H} \oplus \widetilde{\mathbf{C}}^{\ell}=\iota^{*} \mathbf{L}_{1} \oplus \ldots \oplus \iota^{*} \mathbf{L}_{q} .
$$

We will call $\iota^{*} \mathbf{L}_{r}$ a normal or a tangential summand of (2.7) depending on whether it is a summand of the normal bundle or not. This distinction between normal and tangential can also be formulated in terms of the action of $\Gamma$ on $\iota^{*} \mathbf{L}_{r}$. Since $\Gamma$ acts trivially on $Z$, it acts on the line bundles $\mathbf{L}_{i}$ and $\mathbf{L}$ by multiplication by characters of the group, $\Gamma$; and we will denote these characters by $\gamma_{i}$ and $\gamma$.

Lemma 2.1. Let $g$ be an element of $\Gamma^{\#}$. Then $\mathbf{L}_{r}$ is normal or tangential depending on whether $\gamma_{r}(g) \neq 1$ or $\gamma_{r}(g)=1$. 
Proof. If $\gamma_{r}(g)=1, g$ acts trivially on the 2-dimensional subspace of the normal bundle of $Z$ spanned by $\mathbf{L}_{r}$; and hence the fixed point set of $\tau(g)$ in $M$ is strictly larger than $Z$ itself. Thus $g \notin \Gamma^{\#}$. q.e.d.

Let

$$
\mathcal{T}_{F}^{\text {nor }}=\sum_{g \in \Gamma^{\#}} \gamma(g) \prod_{i}^{\prime}\left(1-\gamma_{i}(g)^{-1} \exp \left(-c\left(\mathbf{L}_{i}\right)\right)\right)^{-1}
$$

the superscript "prime" indicating that the product is over those $i$ 's for which $\iota^{*} \mathbf{L}_{i}$ is a normal summand of (2.7), and let

$$
\mathcal{T}_{F}^{\tan }=\exp c(\mathbf{L}) \prod_{i}{ }^{\prime} c\left(\mathbf{L}_{i}\right)\left(1-\exp \left(-c\left(\mathbf{L}_{i}\right)\right)\right)^{-1},
$$

the superscript "double-prime" indicating that the product is over those $i$ 's for which $\iota^{*} \mathbf{L}_{i}$ is a tangential summand of (2.7). Finally let $\mathcal{T}_{F}$ be the product of (2.8) and (2.9).

Definition 2.2. The Kawasaki-Riemann-Roch number of $F^{0}$ is the integral over $F$ of $(\# \Gamma)^{-1} \mathcal{T}_{F}$.

We will say a few words about why this invariant is interesting: Let $W$ be a compact manifold, and $\omega \in \Omega^{2}(W)$ a symplectic form. Suppose $(W, \omega)$ is prequantizable, i.e., suppose there exist a complex line bundle, $\mathbf{L} \rightarrow W$, and a connection on this bundle whose curvature form is $\omega$. Let $J$ be a complex structure on the tangent bundle of $W$ which is compatible with $\omega$. From the data $(\omega, \mathbf{L}, J)$ one gets a "spin- $\mathbf{C}$ structure" on $W$, and the connection on $\mathbf{L}$ enables one to attach to this spin-C structure a "spin-C Dirac operator." We will not attempt to define here either of these objects. See, however, [16, appendix D].

Let $Q$ be the virtual vector space,

$$
Q^{+}-Q^{-}
$$

where $Q^{+}$is the kernel of the spin-C Dirac operator, and $Q^{-}$the cokernel.

Suppose now that one has an action of $G$,

$$
\tau: G \rightarrow \text { symplectomorphisms of } W,
$$

and a compatible action,

$$
\tau_{1}: G \rightarrow \text { automorphisms of } \mathbf{L},
$$


which preserves the connection. If one chooses $J$ to be $G$-invariant (which one can always do) one gets a representation of $G$ on $Q$, and one can compute the weight multiplicities of this representation as follows: The existence of the homomorphism (2.12) implies that the action (2.11) is a Hamiltonian action. In fact the existence of (2.12) not only guarantees that there exists a moment map,

$$
\Phi: W \rightarrow \mathfrak{g}^{*},
$$

but, in fact, via the connection on $\mathbf{L}$, gives one a canonical choice of $\Phi$ (see [15, p.169]).

Theorem 2.2. Let $\mu \in \mathfrak{g}^{*}$ be in the weight lattice of $G$. If $\mu$ is a regular value of the moment map (2.13), the multiplicity with which $\mu$ occurs as a weight of the representation of $G$ on $Q$ is equal to the Kawasaki-Riemann-Roch number of the reduced space

$$
X_{\mu}=: \Phi^{-1}(\mu) / G \text {. }
$$

\section{Remarks}

1. The assumption that $\mu$ is a regular value of $\Phi$ implies that $\Phi^{-1}(\mu)$ is a manifold and that the action of $G$ on this manifold is locally free. Hence, by (2.14), $X_{\mu}$ is an orbifold (and hence $\operatorname{KRR}\left(X_{\mu}\right)$ is well-defined).

2. Special cases of this theorem were proved by us and by Shlomo Sternberg in [9] and [10], and we conjectured that this theorem should be true in the generality stated above. This conjecture has recently been proved, independently, by Eckhard Meinrenken and Michele Vergne. For some other recent results bearing on the conjecture see [7], [11], [6], [17] and [19].

3. In Theorem 2.3 the manifold $W$ need not necessarily be compact. If the moment map, $\Phi$, is proper, this theorem is true providing solutions of the spin-C Dirac operator are appropriately defined at infinity. For instance, for the action of $T$ on $\mathbf{C}^{d}$ discussed in $\S 1$ one should take $Q$ to be the Bargmann space, $\mathcal{B}_{d}$.

\section{Riemann-Roch for toric orbifolds}

We will show below that the Kawasaki-Riemann-Roch number of the orbifold (1.9) is given by an expression of the form (1.4) and explicitly 
compute the $\mathcal{T}_{I}$ 's. Let $\kappa$ be the linear action of the standard $d$-torus, $T^{d}$, on $\mathbf{C}^{d}$ defined by

$$
\kappa\left(e^{i \theta}\right) z=\left(e^{i \theta_{1}} z_{1}, \ldots, e^{i \theta_{d}} z_{d}\right) .
$$

This action preserves the symplectic form (1.7) and is Hamiltonian, its moment map being the map

$$
\Psi(z)=\left(\left|z_{1}\right|^{2}, \ldots,\left|z_{d}\right|^{2}\right)
$$

Moreover it commutes with the action (1.6); so one gets an induced Hamiltonian action of $T^{d}$ on the reduced space (1.10). Letting $J$ be the moment map associated with this action we will prove:

Theorem 3.1. The image of $J$ is the polytope, $\Delta_{\mu+\epsilon}$.

Proof. By (1.10) the image of $J$ coincides with the image under $\Psi$ of the set, $\Phi^{-1}(\mu+\epsilon)$. However, by (1.8) this is the set of all $d$-tuples $\left(z_{1}, \ldots, z_{d}\right), z_{i} \in \mathbf{C}$, satisfying

$$
\left|z_{1}\right|^{2} \alpha_{1}+\ldots+\left|z_{d}\right|^{2} \alpha_{d}=\mu+\epsilon .
$$

Therefore, by (3.2), $\Psi$ maps this set onto the set of non-negative $d$-tuples $\left(s_{1}, \ldots, s_{d}\right), s_{i} \in \mathbf{R}$, satisfying

$$
s_{1} \alpha_{1}+\ldots+s_{d} \alpha_{d}=\mu+\epsilon .
$$

q.e.d.

For $\epsilon$ small, $\mu+\epsilon$ is in general position with respect to $\alpha_{1}, \ldots, \alpha_{d}$; so the subset of $\mathbf{C}^{d}$ defined by (3.3) is a $T$-invariant submanifold of $\mathbf{C}^{d}$, and $T$ acts on this manifold in a locally free fashion. Let us denote this manifold by $M_{\epsilon}$. To describe the orbifold stratification of the spaces (1.10), which we will need to know in order to compute the KawasakiRiemann-Roch number of $X$, we must compute, for each point, $m \in M_{\epsilon}$, the stabilizer group of $m$ in $T$. These groups turn out to be the following: Let $I$ be a subset of $\{1, \ldots, d\}$ and let $M_{\epsilon}^{I}$ be the subset of $M_{\epsilon}$ defined by the equations

$$
z_{i}=0, i \in I
$$

and the inequalities

$$
z_{i} \neq 0, i \in I^{c}
$$


By (1.6) the element, $\exp x$, of $T$ acts trivially on this set iff

$$
\alpha_{i}(x) \in \mathbf{Z}
$$

for $i \in I^{c}$. Hence:

Theorem 3.2. Let $\Gamma_{I}$ be the subgroup of $T$ defined by (3.7). If $m \in M_{\epsilon}^{I}, \Gamma_{I}$ is the stabilizer of $m$.

The group, $\Gamma_{I}$, has a somewhat nicer description than (3.7): From the exponential map, $x \rightarrow \exp x$ one gets an isomorphism

$$
\mathbf{R}^{n} / \mathbf{Z}^{n} \cong T
$$

Let $\mathbf{Z}^{I}$ be the sublattice of $\mathbf{R}^{n}$ defined by

$$
x \in \mathbf{Z}^{I} \Longleftrightarrow \alpha^{i}(x) \in \mathbf{Z} \text { for } i \in I^{c} .
$$

This lattice contains $\mathbf{Z}^{n}$ and under the isomorphism (3.8), $\Gamma_{I}$ gets mapped isomorphically onto the quotient, i.e.,

$$
\Gamma_{I}=\mathbf{Z}^{I} / \mathbf{Z}^{n}
$$

The $M_{\epsilon}^{I}$ 's define a $T$-invariant stratification of $M_{\epsilon}$, and from this stratification, one gets a symplectic stratification of $X_{\epsilon}$ with strata

$$
X_{\epsilon}^{I}=M_{\epsilon}^{I} / T
$$

the structure group of this stratum being $\Gamma_{I}$. An alternative description of this stratification is the following:

Theorem 3.3. The strata, $X_{\epsilon}^{I}$, of $X_{\epsilon}$ are the pre-images with respect to $J$ of the faces, $\Delta_{\mu+\epsilon}^{I}$, of $\Delta_{\mu+\epsilon}$.

This, combined with Theorem 6.4 of [8] implies:

Theorem 3.4. The symplectic volume of $X_{\epsilon}^{I}$ is equal to the volume of the polytope, $\Delta_{\mu+\epsilon}^{I} \cdot{ }^{1}$

\footnotetext{
${ }^{1}$ Euclidean volume in the $(d-n-|I|)$-plane

$$
\sum s_{i} \alpha_{i}=\mu \quad, \quad s_{i}=1 \text { for } i \in I
$$

has been normalized so that the quotient of this plane by the lattice of integer-valued solutions of $\left(^{*}\right)$ has volume $(2 \pi)^{n}$.
} 
Since the symplectic form (1.7) is exact, the symplectic structure on $\mathbf{C}^{d}$ is pre-quantizable. In fact (by [15], loc. cit.) the prequantum line bundle, $\mathbf{L}_{P Q}$, over $\mathbf{C}^{d}$ admits a $T^{d}$-invariant connection and a nonvanishing $T^{d}$-invariant section with covariant derivative:

$$
\nabla s_{0} / s_{0}=2 \pi i \sum_{r=1}^{d} x_{r} d y_{r}-y_{r} d x_{r} .
$$

We will show that when $\epsilon=0$ this pre-quantum structure on $\mathbf{C}^{d}$ induces a pre-quantum structure on the reduced space (1.10): For $\epsilon=0$ let

$$
M_{\epsilon}=M, X_{\epsilon}=X \text {, etc. }
$$

and let $\iota$ be the inclusion of $M$ into $\mathbf{C}^{d}$. Recall that since $X$ is the quotient, $M / T$, there is a branched fibration

$$
\pi: M \rightarrow X
$$

Lemma 3.5. A section, $s$, of $\iota^{*} \mathbf{L}_{P Q}$ is autoparallel along the fibers of $\pi$ iff it transforms under $T$ according to the character

$$
\gamma_{\mu}(\exp x)=e^{2 \pi i \mu(x)}
$$

Proof. By (1.6) the infinitesimal action of $T$ on $\mathbf{C}^{d}$ is given by

$$
v_{M}=\sum \alpha_{i}(v)\left(x_{i} \frac{\partial}{\partial y_{i}}-y_{i} \frac{\partial}{\partial x_{j}}\right)
$$

for $v \in \mathfrak{t}=\mathbf{R}^{n}$. From (3.12) it follows that

$$
\nabla_{v_{M}} s_{0}=\left(\sum \alpha_{i}(v)\left|z_{i}\right|^{2}\right) s_{0}=\mu(v) s_{0},
$$

so that if $s=f s_{0}$, and $s$ is autoparallel along the fibers of $\pi$, then

$$
D_{v_{M}} f=-\mu(v) \text {. }
$$

Hence $f$ has to transform under $T$ according to the character, $\gamma_{\mu}$.

q.e.d.

The line bundle, $\iota^{*} \mathbf{L}_{P Q}$, is not necessarily the pull-back of a line bundle on $X$. However, the autoparallel sections of $\iota^{*} \mathbf{L}_{P Q}$ are the sections of a rank-one sheaf, and we will, by abuse of language, refer to the 
sections of this sheaf as sections of the "pre-quantum line bundle" on $X$. If $s$ is a section of $\iota^{*} \mathbf{L}_{P Q}$, which is autoparallel along the fibers of $\pi$, and $v$ is a $T$-invariant vector field, then $\nabla_{v} s$ is also autoparallel along the fibers of $\pi$, so this "pre-quantum line bundle" has a well-defined connection.

To apply the results of Section 2 to $X$ we will need another description of this pre-quantum line bundle: We will need a line bundle, $\mathbf{L}_{\mu} \rightarrow M$, and an action of $T$ :

$$
\tau_{0}: T \rightarrow \operatorname{aut}\left(\mathbf{L}_{\mu}\right)
$$

which is compatible with the action (1.6), such that the sheaf of $T$ invariant sections of $\mathbf{L}$ coincides with the sheaf of sections of the prequantum line bundle on $X$. By Lemma 3.5, however, one can simply take $\mathbf{L}$ to be the trivial bundle

$$
\widetilde{\mathbf{C}}=M \times \mathbf{C}
$$

and define $\tau_{0}$ by requiring that the constant section of (3.17) transforms according to the character, $\gamma$.

Let $\mathbf{L}_{r}$ be another copy of the trivial bundle (3.17) and let

$$
\tau_{r}: T \rightarrow \operatorname{aut}\left(\mathbf{L}_{r}\right)
$$

be defined by the property that the constant section of (3.17) transforms under $\tau_{r}$ according to the character

$$
\gamma_{r}(\exp x)=e^{2 \pi i \alpha_{r}(x)}, r=1, \ldots, d .
$$

We claim that, in analogy with (2.4), there is an equivariant splitting

$$
T^{H} M \oplus \widetilde{\mathbf{C}}^{n}=\mathbf{L}_{1} \oplus \ldots \oplus \mathbf{L}_{d} \text {. }
$$

Proof. By (1.6) the restriction of the tangent bundle of $\mathbf{C}^{d}$ to $M$ is equal to the right-hand side of $(3.20)$. On the other hand, since $\mu$ is a regular value of $\Phi$ and $M=\Phi^{-1}(\mu)$, the normal bundle to $M$ in $\mathbf{C}^{d}$ can be identified with $\sqrt{-1} \widetilde{\mathbf{R}}^{n}$, and the vertical component of the tangent bundle of $M$ can be identified with $\widetilde{\mathbf{R}}^{n}$, so the restriction of the tangent bundle of $\mathbf{C}^{d}$ to $M$ can also be identified equivariantly with the left-hand side of (3.20). q.e.d.

Let

$$
\widetilde{c}\left(\mathbf{L}_{i}\right) \in H_{G}^{2}(M, \mathbf{C})
$$


be the equivariant Chern classes of the line bundles, $\mathbf{L}_{i}$, and let

$$
c_{i}=c\left(\mathbf{L}_{i}\right), i=1, \ldots, d
$$

be their images in $H^{2}(X, \mathbf{C})$. We will describe how these Chern classes are related to the Chern classes

$$
c_{r}^{\#} \in H^{2}(X, C), \quad r=1, \ldots, n
$$

of the principal bundle, $M \rightarrow X .^{2}$ Let $v^{1}, \ldots, v^{n}$ be the standard basis vectors of $\mathbf{R}^{n}$ ( $\mathbf{R}^{n}$ being the Lie algebra of $T$ ) and let $e^{1}, \ldots, e^{d}$ be the standard basis vectors of $\mathbf{R}^{d}$. By (1.6) $T$ imbeds in $T^{d}$. Let

$$
\iota: \mathbf{R}^{n} \rightarrow \mathbf{R}^{d}
$$

be the corresponding imbedding of Lie algebras. By (1.6) the dual map, $\iota^{*}$, is the map

$$
\iota^{*}\left(e_{r}\right)=\alpha_{r}
$$

the $e_{r}$ 's being the dual basis vectors in $\left(\mathbf{R}^{d}\right)^{*}$ to the $e^{r}$ 's. We claim

$$
\sum_{r=1}^{n} c_{r}^{\#} \iota\left(v^{r}\right)=\sum_{i=1}^{d} c_{i} e^{i} .
$$

Proof. Since $\mathbf{L}_{k}$ is the line bundle associated to the principal bundle, $M \rightarrow X$, by means of the character (3.19), its Chern class is

$$
c\left(\mathbf{L}_{k}\right)=\sum \alpha_{k}\left(v_{r}\right) c_{r}^{\#}
$$

so the right-hand side of $(3.25)$ is

$$
\sum_{r} c_{r}^{\#}\left(\sum_{k} \alpha_{k}\left(v^{r}\right) e^{k}\right) .
$$

However, by (3.24), the expression in the parentheses is $\iota\left(v^{r}\right)$. q.e.d.

Recall that the group, $\Gamma_{I}$, is the subgroup of $T$ defined by the equalities

$$
\gamma_{i}(g)=1, i \in I^{c}
$$

\footnotetext{
${ }^{2}$ Since the action of $T$ on $M$ is merely "locally free" there should perhaps be quotation marks about "principal".
} 
Let $\Gamma_{I}^{\#}$ be the subset of $T$ defined by the equalities (3.26) and the inequalities

$$
\gamma_{i}(g) \neq 1, i \in I
$$

We will define the "Todd function" $\mathcal{T}_{I}\left(x_{1}, \ldots, x_{d}\right)$ to be the function:

$$
\left(\# \Gamma_{I}\right)^{-1}\left(\prod_{i \in I^{c}} x_{i}\right) \sum_{g \in \Gamma_{I}^{\#}} \gamma_{\mu}(g) \prod_{i=1}^{d}\left(1-\gamma_{i}(g)^{-1} e^{-x_{i}}\right)^{-1}
$$

where the factor in front of the summation sign is a "counter-term" introduced to cancel the infinities caused by the fact that, by (3.26), $\gamma_{i}(g)=1$ when $i \in I^{c}$. This function is regular at $x=0$; so we can expand it in a Taylor series. We will denote by

$$
\mathcal{T}_{I}\left(\frac{\partial}{\partial \epsilon_{1}}, \ldots, \frac{\partial}{\partial \epsilon_{d}}\right)
$$

the "infinite-order" constant coefficient differential operator which one gets by substituting $\frac{\partial}{\partial \epsilon_{i}}$ for $x_{i}$ in this Taylor series. We claim that, with this definition of the $\mathcal{T}_{I}$ 's, the Kawasaki-Riemann-Roch number of $X$ is given by the right-hand side of (1.4).

Proof. Let $\epsilon=\sum \epsilon_{i} \alpha_{i}$. By the Duistermaat-Heckman theorem and (3.25), the symplectic volume of $X_{\epsilon}^{I}$ is equal to

$$
\int_{X^{I}} \exp \left(\left[\omega_{\text {red }}\right]+\sum_{i=1}^{d} \epsilon_{i} c_{i}\right) \text {. }
$$

This expression is a polynomial in $\epsilon_{1}, \ldots, \epsilon_{d}$; so we can apply the infinite-order differential operator, $\mathcal{T}_{I}$ to it. Setting $\epsilon=0$ this gives us

$$
\int_{X^{I}} \exp \left[\omega_{\text {red }}\right] \mathcal{T}_{I}\left(c_{1}, \ldots, c_{d}\right),
$$

which, by (3.28) and (2.8)-(2.9) is the Kawasaki-Riemann-Roch number of $X^{I}$. On the other hand, by Theorem 3.4, the expression (3.30) is equal to the volume of the polytope, $\Delta_{\mu+\epsilon}^{I}$, so (3.31) is equal to

$$
\mathcal{T}_{I}\left(\frac{\partial}{\partial \epsilon}\right) v^{I}(\epsilon)
$$

evaluated at $\epsilon=0$. Summing these expressions over $I$, we get for the Kawasaki-Riemann-Roch number of $X$ the right-hand side of (1.4). 


\section{Equivariant Kawasaki-Riemann-Roch}

Let $X$ be a compact pre-quantizable symplectic orbifold, and let $\rho: T^{d} \rightarrow \operatorname{Diff}(X)$ be a Hamiltonian action of the torus, $T^{d}$, on $X$. The equivariant Kawasaki-Riemann-Roch number of $X$ is defined, as in $\S 2$, to be the sum, over the strata, $F$, of $X$, of the integrals

$$
(\# \Gamma)^{-1} \int_{F} \mathcal{J}_{F}^{\text {tan }} \cdot \mathcal{J}_{F}^{\text {nor }}
$$

where $\mathcal{J}_{F}^{\text {tan }}$ and $\mathcal{J}_{F}^{\text {nor }}$ are defined as in $\S 2$ by the expressions $(2.8)$ and (2.9) with one small modification. In (2.8) and (2.9) one has to replace $c(\mathbf{L})$ and $c_{i}(\mathbf{L})$ by their equivariant counterparts, which are, by definition, elements of $H_{T^{d}}^{2}(X)$. Thus the integral (4.1) is no longer just a number. It is an element of the formal power series ring, $\mathbf{C}\left[\left[\xi_{1}, \ldots, \xi_{d}\right]\right]$.

Suppose now that the manifold, $W$, figuring in Theorem 2.3 is equipped with a Hamiltonian action of $T^{d}$ which commutes with the action of $G$. One then gets an induced Hamiltonian action of $T^{d}$ on the space (2.14) and induced representations of $T^{d}$ on each of the weight spaces, $Q^{\mu}$, of $Q$; the equivariant version of Theorem 2.3 states:

Theorem 4.1. Let $\gamma$ be the character of the representation of $T^{d}$ on $Q^{\mu}$. Then the Taylor series of $\gamma(\exp \xi)$ at $\xi=0$ is equal to the equivariant Kawasaki-Riemann-Roch "number" of $X_{\mu}$.

Let us apply this result to the representation of $T^{d}$ on the Bargmann space, $\mathcal{B}_{d}$. We pointed out in $\S 1$ that the vectors in $\mathcal{B}_{d}$ which transform under $T$ according to the weight, $\mu$, are just the monomials

$$
z_{1}^{k_{1}} \ldots z_{d}^{k_{d}}
$$

for which $\left(k_{1}, \ldots, k_{d}\right)$ is a solution of (1.1). Thus the character of the representation of $T^{d}$ on this space is

$$
\sum e^{i \xi \cdot k}
$$

summed over the solutions, $k$ of (1.1). On the other hand, the equivariant Kawasaki-Riemann-Roch number of the reduced space (1.9) is, by (3.30) and (3.31), equal to the sum over the multi-indices, $I$, of the expressions

$$
\mathcal{T}_{I}\left(\frac{\partial}{\partial \epsilon}\right) \int_{X^{I}} \exp \left(\left[\widetilde{\omega}_{\text {red }}\right]+\sum \epsilon_{i} \widetilde{c}_{i}\right)
$$


evaluated at $\epsilon=0, \widetilde{\omega}_{\text {red }}$ being the equivariant reduced symplectic form on $X$, and the $\widetilde{c}_{i}$ 's being the equivariant Chern classes of $X$. However, by the Duistermaat-Heckman theorem, $\left[\widetilde{\omega}_{\text {red }}\right]+\sum \epsilon_{i} \widetilde{c}_{i}$ is the cohomology class of the equivariant symplectic form on the reduced space (1.10). This form can also be written as

$$
\omega_{\mathrm{red}}^{\epsilon}+\sqrt{-1} \sum \xi_{i} \phi_{i}^{\epsilon}
$$

where $\omega_{\text {red }}^{\epsilon}$ is the usual reduced symplectic form on the space (1.10), and the $\phi_{i}^{\epsilon}$ 's are the coefficients of the $T^{d}$-moment map. Thus (4.3) is equal to the operator $\mathcal{T}_{I}\left(\frac{\partial}{\partial \epsilon}\right)$ applied to the expression

$$
\int_{X_{\epsilon}^{I}}\left(\exp \omega_{\mathrm{red}}^{\epsilon}\right) e^{\sqrt{-1} \sum \xi_{i} \phi_{i}^{\epsilon}} \text {. }
$$

However, by the measure-theoretic version of Duistermaat-Heckman this is equal to

$$
\int_{\Delta_{\mu+\epsilon}^{I}} e^{\sqrt{-1} \sum \xi_{i} s_{i}} d s,
$$

so the sum over the $I$ 's of (4.3) reduces to (1.15).

\section{Meinrenken-Sjamaar}

As in $\S 2$ let $W$ be a compact manifold, $\omega \in \Omega^{2}(W)$ a symplectic form and $\mathbf{L}$ a complex line bundle on $W$ whose Chern class in [ $\omega$ ]. Let $G$ be an $n$-torus and let $G$ act on $\mathbf{L}$ by bundle morphisms so that the induced action on $W$ preserves $\omega$. One then gets an induced representation of $G$ on the space (2.10); and for any integer lattice point, $\mu$, in $\mathfrak{g}^{*}$ which is a regular value of the moment map (2.13), the multiplicity with which $\mu$ occurs as a weight of this representation is equal, by Theorem 2.2 , to the Kawasaki Riemann-Roch number of the orbifold (2.14). If $\mu$ is not a regular value of $\Phi$, then $X_{\mu}$ is not an orbifold; however, it is a stratified space whose strata are symplectic manifolds satisfying the Goresky-MacPherson axioms of intersection cohomology. (C.f. [23].) In particular one can define mixed characteristic numbers (in intersection cohomology) for $X_{\mu}$ analogous to the integrals (1.12). Moreover, even though $X_{\mu}$ is not a manifold (or orbifold), its local structure can be described by canonical form theorems analogous to the equivariant Darboux theorems for orbifolds (loc. cit.). Using these results Meinrenken 
and Sjamaar have recently shown that $X_{\mu}$ can be desingularized in a more or less intrinsic way, and that one can unambiguously define the Riemann-Roch number of $X_{\mu}$ as the Kawasaki Riemann-Roch number of this desingularization. They have also shown that this Riemann-Roch number can be computed (in a slightly less intrinsic way) by means of the moment map, $\Phi$. More explicitly, for $\epsilon$ small, let $\mu+\epsilon$ be a nontrivial regular value of $\Phi$ (i.e., a regular value which actually lies in the image of $\Phi)$. Let

$$
X_{\mu+\epsilon}=\Phi^{-1}(\mu+\epsilon) / G,
$$

and let $c_{i} \in H^{2}\left(X_{\mu+\epsilon}\right), i=1, \ldots, n$, be the Chern clases of the fibration

$$
G \longrightarrow \Phi^{-1}(\mu+\epsilon) \longrightarrow X_{\mu+\epsilon} .
$$

Finally define $c(\mathbf{L})$ to be the cohomology class

$$
\omega_{\epsilon}-\sum \epsilon_{i} c_{i}
$$

where $\omega_{\epsilon}$ is the reduced symplectic form on $X_{\mu+\epsilon}$, and let $\gamma: G \longrightarrow$ $S^{1}$ be the character (3.14). Meinrenken and Sjamaar prove that the intrinsically defined Riemann-Roch number of $X_{\mu}$ which we alluded to above is equal to the sum

$$
\sum_{F}\left(n_{F}\right)^{-1} \int_{F} \mathcal{T}_{F}
$$

over the orbifold strata of $X_{\mu+\epsilon}, n_{F}$ being the cardinality of the isotropy group of $F$, and $\mathcal{T}_{F}$ being defined by (2.8)-(2.9), with $c(\mathbf{L})$ and $\gamma$ defined as above. In particular the sum (5.4) is independent of $\epsilon$. They go on to prove that Theorem 2.2 remains true for reduction at singular values of the moment map:

Theorem 5.1. The Riemann-Roch number (5.4) is equal to the multiplicity with which $\mu$ occurs as a weight of the representation of $G$ on the space (2.10).

For details see: E. Meinrenken and R. Sjamaar, "Riemann-Roch formulas for multiplicities and singular reduction," (pre-print MIT, September, 1995). By applying their result to the action of $G$ on $\mathcal{B}_{d}$ described in $\S 1$, one obtains the recipe (1.17) for computing the number of solutions of the equation (1.1) for $\mu$ not in general position with respect to the $\alpha_{i}$ 's. 


\section{References}

[1] R. Bott \& L. Tu, Differential forms in algebraic topology, Springer, New York, 1982.

[2] M. Brion, Points entiers dans les polyèdres convexes, Ann. Sci. École Norm. Sup. 21 (1988) 653-665.

[3] M. Brion \& M. Vergne, Lattice points in simple polytopes, Preprint, May, 1995.

[4] S. Cappell \& J. Shaneson, Characteristic classes, lattice points and Euler-Maclaurin formulae, Proc. Internat. Congr. Math. (Zurich, 1996), to appear.

[5] V.I. Danilov, The geometry of toric varieties, Russian Math. Surveys 33 (1978) 97-154.

[6] J.J. Duistermaat, V. Guillemin, E. Meinrenken \& S. Wu, Symplectic reduction and Riemann-Roch for circle actions, Math. Res. Lett. 2 (1995) 259-266.

[7] V. Guillemin, Reduced phase spaces and Riemann-Roch, in Lie Theory and Geometry, Progr. Math. 123 (1994) 305-334.

[8] __ Kaehler structures on toric varieties, J. Differential Geom. 40 (1994) 285309.

[9] V. Guillemin \& S. Sternberg, Geometric quantization and multiplicities of group representations, Invent. Math. 67 (1982) 515-538.

[10] Homogeneous quantization and multiplicities of group representations, J. Funct. Anal. 47 (1982) 344-380.

[11] L. Jeffrey \& F. Kirwan, On localization and Riemann-Roch numbers for symplectic quotients, Preprint, September, 1994.

[12] T. Kawasaki, The Riemann-Roch theorem for complex V-manifolds, Osaka J. Math. 16 (1979) 151-159.

[13] A. Khovanskii \& J.M. Kantor, Integral points in convex polyhedra, combinatorial Riemann-Roch and generalized Maclaurin formulae, Inst. Hautes Études Sci. Publ. Math. (1992) 932-937.

[14] A. Khovanskii \& S. Pukhlikov, Théorème de Riemann-Roch pour les intégrales et les sommes de quasi-polynômes sur les polyèdres virtuels, Algebra i analiz 4 (1992) 188-216.

[15] B. Kostant, Quantization and unitary representations, Lecture notes in Math. Vol. 170, Springer, Berlin, 1970, 87-207.

[16] B. Lawson \& M. Michelsohn, Spin Geometry, Princeton Univ. Press, Princeton, 1989.

[17] S. Martin \& J. Weitsman, On a conjecture of Guillemin-Sternberg, to appear. 
[18] E. Meinrenken, On Riemann-Roch formulas for multiplicities, J. Amer. Math. Soc., to appear.

[19] - Symplectic surgery and the spin ${ }^{c}$-Dirac operator, Preprint, March, 1995.

[20] R. Morelli, Pick's theorem and the Todd class of a toric variety, Adv. Math. 100 (1993) 183-231.

[21] J.E. Pommersheim, Toric varieties, lattice points and Dedekind sums, Math. Ann. 295 (1993) 1-24.

[22] I. Satake, On a generalization of the notion of manifold, Proc. Nat. Acad. Sci. U.S.A. 42 (1956) 359-363.

[23] R. Sjamaar \& E. Lerman, Stratified symplectic spaces and reduction, Ann. of Math. 134 (1991) 375-422.

[24] B. Sturmfels, On vector partition functions, Preprint, July, 1994.

[25] M. Vergne, Quantification géométrique et multiplicités, C.R. Acad. Sci. Paris, 319 (1994) 327-332.

MASSACHUSETTS INSTITUTE OF TECHNOLOGY 